\title{
EFECTOS DE DIFERENTES MÉTODOS DE CALENTAMIENTO UTILIZADOS EN EL PERIOPERATORIO EN EL ADULTO
}

\author{
EFFECTS OF DIFFERENT METHODS USED IN PERIOPERATIVE \\ WARMING IN THE ADULT
}

\author{
Claudia Verónica Pérez Acuña* \\ Angélica Ivonne Cerda Gallardo** \\ Viviana Andrea Munilla GonZÁlez ${ }^{* *}$
}

\begin{abstract}
RESUMEN
Antecedentes: $\mathrm{Al}$ menos un 50\% de los pacientes quirúrgicos presentan algún grado de hipotermia. Esta se ha asociado a diversos efectos adversos metabólicos, hematológicos, cardiovasculares y farmacológicos, entre otros. Objetivo: conocer el efecto del calentamiento durante el periodo perioperatorio en pacientes adultos. Metodología: revisión de literatura que recopila 22 investigaciones randomizadas controladas, 11 revisiones bibliográficas, 3 estudios prospectivos, 1 metanálisis, 1 revisión sistemática y 1 guía clínica, publicadas entre enero de 1996 y octubre de 2006, obtenidos desde distintas bases de datos y revistas científicas. Resultados: existen diversos sistemas de calentamiento, siendo el más utilizado y efectivo el aire caliente forzado. El calentamiento es utilizado en todo tipo de cirugías (excepto en las neurológicas y cardiacas), principalmente durante el intraoperatorio. Los principales efectos en la etapa preoperatoria son el aumento de la temperatura corporal y la facilitación de la canulación venosa. En la etapa intraoperatoria produce mantención de la normotermia y disminución en el sangramiento durante la cirugía, ambos efectos se mantienen durante la etapa postoperatoria. En la última fase del perioperatorio, es posible observar una reducción de la infección de la herida operatoria, escalofríos, vasoconstricción, presión arterial, eventos mórbidos cardíacos, tiempo de ventilación mecánica, uso de vasodilatadores, morfina y antibióticos, y un aumento del confort térmico. Conclusiones: se respalda la aplicación del calentamiento como un método efectivo, ya sea previniendo o tratando la hipotermia perioperatoria inadvertida y sus complicaciones, lo que reduce el tiempo de recuperación, los costos de la hospitalización y aumenta el confort térmico.
\end{abstract}

Palabras clave: Calentamiento, cirugía, hipotermia, perioperatorio, temperatura.

\begin{abstract}
Background: At least 50\% surgical patients have some hypothermia degree. In Addition, hypothermia may have several metabolic, haematologic, cardiovascular and pharmacological adverse consequences, between other things. Aim: it is to know the warming effects during perioperative in surgical adults patients. Methods: literature review of twenty two randomized controlled trials, eleven literature review, three prospective trials, one metaanalysis, one systematic review, and one clinical guideline, published between January 1996 and October 2006, all this evidence have been got of different data bases and scientific magazine. Results: randomized controlled trials show the application of several warming systems, the most used and effective is air forced. Warming used in all types of surgery (except neurological and cardiac surgery), mainly during intraoperative. The principal effects of warming in the preoperative are: increase patient temperature, and facilitate venous cannulation. During intraoperative mantains normothermia and decrease blood loss during surgery, both effects remain in the postoperative. Furthermore in the last perioperative phase is possible found a reduce of wound infection,
\end{abstract}

\footnotetext{
*Enfermera Matrona, Especialista en Enfermería Nefrourológica, Magíster en Psicología, docente Carrera de Enfermería Universidad del Desarrollo. E-mail:claudiaperez@udd.cl

${ }^{* *}$ Enfermera Matrona, Enfermera Clínica UC San Carlos de Apoquindo. E-mail:aicerda@uc.cl

${ }^{* * *}$ Enfermera Matrona, Enfermera Clínica UC San Carlos de Apoquindo. E-mail: vamunill@uc.cl
} 
hospital stay, shivering, vasoconstriction, blood pressure, morbid cardiac events, mechanical ventilation time, vasodilators, morphine and antibiotics use, pain, and increase thermal comfort. Conclusion: this evidence review provide support that warming is an effective method, preventing or treatment unplanned perioperative hypothermia and its adverse consequences, reducing recuperation time, hospitalization costs and increasing thermal comfort.

Key words: Hypothermia, perioperative, temperature, surgery, warming.

Fecha recepción: 20/08/07 Fecha aceptación: 30/10/09

\section{INTRODUCCIÓN}

El proceso quirúrgico supone diversos riesgos para el usuario, entre ellos, la hipotermia, al menos un $50 \%$ de los pacientes quirúrgicos presentan algún grado de hipotermia, $y$ un $44 \%$ corresponde a hipotermia intraoperatoria (1). La disminución de la temperatura podría afectar al $70 \%$ de los pacientes al ingreso en la unidad de recuperación (2).

La hipotermia se ha asociado a diversos efectos adversos en el periodo postoperatorio tales como: disminución del metabolismo, aumento en la incidencia de infección de la herida operatoria y retardo en la cicatrización, mayor pérdida de sangre y requerimiento de fluidos postoperatorios, hipertensión, isquemia miocárdica y arritmias cardiacas, disminución del metabolismo de los fármacos, escalofríos, disconfort térmico, incrementa la necesidad de ventilación mecánica postoperatoria, e incluso aumenta la mortalidad. Todas estas complicaciones se traducen en un aumento del tiempo de hospitalización y por lo tanto de los costos ( 1 , 3-7).

Se han descrito variadas técnicas para minimizar la hipotermia (2), éstas se clasifican en pasivas y activas (8). Frente a la hipotermia que se puede presentar en el perioperatorio y a la aplicación de diversos sistemas de calentamiento, surge la siguiente interrogante ¿Cuál es el efecto del calentamiento durante el periodo perioperatorio en el adulto sometido a cirugía?

Objetivo general: Conocer la evidencia que existe en relación al efecto de diversos sistemas de calentamiento durante el periodo perioperatorio en pacientes adultos.

Objetivos específicos: Identificar y describir los diferentes sistemas de calentamiento utilizados en el periodo perioperatorio, reconocer cómo afecta el calentamiento durante el periodo perioperatorio en pacientes adultos, identificar los beneficios y desventajas de la aplicación del calentamiento en el período perioperatorio y sus consecuencias, determinar la implicancia para enfermería que tiene conocer el efecto del calentamiento durante el periodo perioperatorio en el paciente adulto.

\section{MATERIAL Y MÉTODO}

La revisión de la literatura se realizó a partir de la búsqueda de artículos, encontrándose 22 investigaciones randomizadas controladas, 11 revisiones bibliográficas, 3 estudios prospectivos, 1 metanálisis, 1 revisión sistemática y 1 guía clínica. Se utilizaron bases de datos (Proquest Nursing Journals, Pubmed, Scopus, Silverplatter, Medline, Scielo, The Cochrane library, Lilacs y Cinhal), y revistas impresas-electrónicas (Journal of advances nursing, Advances in nursing science, American Journal of Medicine, American journal of nursing, American journal of surgery, Anesthesia \& Analgesia, Anesthesiology, Applied nursing research, Best practice and research clinical anesthesiology, British journal of anesthesia, Canadian Journal of anes- 
thesia, Controlled Clinical trials, Current anaesthesia and critical care). Se limitó la búsqueda a bibliografía publicada entre enero de 1995 y octubre de 2006, escritos en inglés o español y de texto completo. Se utilizaron las siguientes palabras clave: anesthesia, hypothermia, perioperative, temperature, surgery, warming, y sus análogos en español.

\section{Sistemas de calentamiento perioperatorio en el paciente adulto}

Los sistemas y medidas de calentamiento se pueden clasificar en dos grandes grupos: activos y pasivos (8). El calentamiento pasivo incluye el aumento de la temperatura del pabellón, y cubrir las superficies expuestas utilizando los paños quirúrgicos, mantas de algodón o de aluminio, las que limitan las pérdidas por radiación y convección $(5,9)$.

Actualmente empresas internacionales ofrecen diversos sistemas de calentamiento, éstos se denominan activos ya que no sólo evitan la pérdida de calor, sino que también aportan calor. Forman parte de este grupo las lámparas radiantes o infrarrojos, las mantas eléctricas, los colchones o mantas por los que circula agua caliente, el aire caliente convectivo, calentamiento de fluidos intravenosos y de irrigación, calentamiento y humidificación de los gases anestésicos y calentadores de $\mathrm{CO}_{2}$ para cirugía laparoscópica $(2,3,8,9)$.

En los estudios revisados, respecto a los sistemas de calentamiento mencionados anteriormente, se puede apreciar que el aire caliente forzado y el dispositivo de calentamiento de fluidos se utilizan con mayor frecuencia, lo que concuerda con las recomendaciones de la guía clínica para la prevención de la hipotermia perioperatoria inadvertida del 2001 (10).

Respecto a la indicación de los sistemas de calentamiento, éstos son aplicados especialmente en procedimientos quirúrgicos abdominales y ortopédicos, con una menor frecuencia en cirugías cardiacas, plásticas, ginecológicas, vasculares, torácicas, otorri- nolaringológicas, mamarias, urológicas y procedimientos con duración superior a 2 horas.

\section{EFECTOS DEL CALENTAMIENTO EN EL PERIOPERATORIO}

A continuación se presentan los efectos del calentamiento, según la etapa perioperatoria.

\section{Período preoperatorio}

De acuerdo a las investigaciones, el único efecto de la aplicación del calentamiento observado durante este periodo es el aumento de la temperatura previa a la cirugía (11-16). Se realizó un estudio para determinar si el calentamiento facilita la canulación venosa periférica. Demostrando éxito para la inserción de la cánula en un $94 \%$ en el grupo de calentamiento y el tiempo promedio de cateterización exitosa se redujo significativamente a la mitad en el grupo de calentamiento activo (14).

\section{Período intraoperatorio}

La aplicación de calentamiento durante este periodo resulta en la mantención de la normotermia desde aproximadamente $15 \mathrm{mi}$ nutos de iniciada su aplicación hasta el final de la cirugía $(11,12,14,16-19)$. Otro efecto reportado durante este periodo es la disminución del sangramiento y la necesidad de transfusiones sanguíneas (20). Estos resultados concuerdan con los obtenidos en un metanálisis (21).

\section{Período postoperatorio}

La normotermia producida por el calentamiento intraoperatorio, se mantiene hasta 5 horas postoperatorias $(11,12,14,16-19)$. En pacientes sometidos a cirugía cardiaca, en los que se aplica el calentamiento desde el final de la cirugía y hasta el cierre de la piel, la temperatura central se mantiene más alta 
hasta por 4 horas (22). Las investigaciones demuestran que el calentamiento durante los periodos pre e intraoperatorio, tanto local como sistémico, reducen significativamente la incidencia de infección de la herida operatoria $(13,15,21)$.

Los escalofríos postoperatorios son una seria complicación de la hipotermia que incrementa el consumo de oxígeno entre un 200 y $600 \%$ y es proporcional a la pérdida de calor durante el periodo intraoperatorio (18). Al respecto, la evidencia muestra que el calentamiento intraoperatorio disminuye los escalofríos postoperatorios en aproximadamente un $30 \%(11,20)$. Se ha estudiado que los pacientes que recibieron calentamiento calificaron este periodo como confortable y no observaron la presencia de sudoración entre ellos (11). En relación a lo anterior, se ha reportado que pacientes que son sometidos a calentamiento refieren mayor confort térmico y ausencia de sensación de frío $(12,14)$.

$\mathrm{Al}$ aplicar calentamiento con aire forzado desde el final de la cirugía hasta el cierre de la piel, en pacientes sometidos a cirugía cardiaca (bypass de arteria coronaria, recambio valvular) se encontró que en estos pacientes, la pérdida de sangre por el drenaje torácico fue significativamente menor en un $31 \%$. Lo que se correlaciona con una reducción en un $23 \%$ en los requerimientos de sueros (22).

Estos resultados se pueden explicar con la investigación realizada en pacientes sometidos a cirugía plástica electiva, en la que se encontró que los pacientes que se mantuvieron hipotérmicos presentaron un TTPK mayor y un tiempo de sangría más largo en comparación con el grupo al que se le aplicó calentamiento intraoperatorio (17).

Respecto a los parámetros hemodinámicos, el calentamiento produce una disminución en la presión arterial diastólica, sistólica y media en el postoperatorio inmediato. Una disminución de $1.5^{\circ} \mathrm{C}$ en la temperatura central se asoció a concentraciones de norepinefrina más altas en el periodo postoperatorio inmediato, lo que podría representar un componente termorregulatorio en la respuesta de esta hormona (23).

Diversos estudios han demostrado que el calentamiento intraoperatorio disminuye la vasoconstricción postoperatoria en aproximadamente un $30 \%$ y este efecto se mantiene por una hora postcirugía $(20,23)$. Esto da cuenta que la normotermia evitaría la activación del sistema nervioso simpático, y por lo tanto el incremento en los niveles de presión arterial.

También se ha observado una incidencia significativamente menor de eventos al electrocardiograma (isquemia y taquicardia ventricular) y de eventos mórbidos cardíacos (angina/isquemia inestable, paro cardiaco e infarto al miocardio) en el periodo postoperatorio, en los pacientes que recibieron calentamiento con aire forzado (23). Estos resultados concuerdan con lo observado en un metanálisis (21). Además demuestran que la mantención de la normotermia perioperatoria se asocia a una disminución de la incidencia de eventos cardiacos en el periodo postoperatorio inmediato (23).

El calentamiento disminuye significativamente los requerimientos de ventilación mecánica de un 18 a un $11 \%(21,23)$. Así mismo, las investigaciones muestran que el calentamiento favorece la extubación endotraqueal precoz $(11,24)$.

El calentamiento cutáneo agresivo esta asociado a una disminución de los requerimientos en la terapia vasodilatadora después de la cirugía $(25,26)$. También se encontró que el calentamiento disminuyó los requerimientos de vasodilatadores en un 13\% (22) y la disminución estadísticamente significativa en el uso de antibióticos postoperatorios de un 15,9 a un $6,5 \%$ (13).

El "score" de dolor postoperatorio fue significativamente menor al aplicar dos horas de calentamiento postoperatorio (15). También se describió la utilización de una cantidad de morfina significativamente menor durante las primeras 12 horas postoperatorias, en las personas que recibieron calentamiento (27). 


\section{Efectos adversos}

Si el calentamiento preoperatorio se aplica de acuerdo a las indicaciones de los fabricantes del dispositivo, no debería causar efectos adversos (15). Dos investigaciones en este sentido refieren que no se presentaron complicaciones atribuibles al calentamiento (14, 19).

\section{Tiempo de hospitalización}

En pacientes sometidos a resección colorrectal, la duración de la hospitalización se redujo en 2,6 días (aproximadamente el 20\%) en los pacientes que se mantuvieron normotérmicos durante la cirugía $(9,21)$. Además se encontró una disminución de aproximadamente un $50 \%$ en la duración de la hospitalización en la unidad de recuperación (18, 20, 24).

\section{Costos}

Al comparar los costos del uso del dispositivo de aire caliente forzado, con la unidad de calor convectivo y las frazadas eléctricas, se encontró que la frazada eléctrica es la más económica (1.542 pesos) y el sistema de aire caliente forzado el más costoso (12.747 pesos) (27).

Al calcular los costos de hospitalización de los pacientes que se sometían a cirugía abdominal mayor se encontró que en los pacientes en los que se aplicó calentamiento activo 30 minutos antes de la inducción anestésica y durante la cirugía, los costos hasta el alta de la unidad de recuperación disminuyeron en 86.814 pesos (20).

Respecto a los costos potenciales del uso del calentamiento, los resultados sugieren que después de 5 años cuando se amortizaron los costos de los dispositivos de calentamiento, éstos eran menores que los costos de las frazadas adicionales utilizados en la unidad de recuperación. Además las unidades de calentamiento son frecuentemente entregadas sin costos a los hospitales. Si se asume que los costos hospitalarios son variables, al comparar los gastos adicionales por la utili- zación de la técnica de calentamiento con la medición de ahorros por mano de obra y suministros, el uso de la técnica disminuye los costos totales para la institución (24).

Al comparar los costos del uso del dispositivo de aire caliente forzado versus la frazada reflectante en pacientes sometidos a cirugía de rodilla, observaron que el aire caliente forzado es significativamente más costoso en comparación con la frazada reflectante, sin embargo, debido a la disminución en el tiempo de hospitalización en la unidad de recuperación, el calentamiento con aire caliente forzado fue significativamente más eficiente y costo efectivo en la mantención de la normotermia perioperatoria durante cirugías de rodilla. Aunque los costos del aire caliente forzado pueden ser mayores que los otros métodos, es compensado por el menor tiempo de permanencia en la sala de recuperación (10.520 pesos por 15 minutos) y el costo por tratamientos asociado a complicaciones por hipotermia postoperatoria $(28,29)$.

\section{CONCLUSIONES}

La aplicación del calentamiento es una medida eficaz (eficiente y efectiva), ya que aumenta la temperatura corporal y el confort térmico, la satisfacción del paciente y facilita la canulación venosa, disminuye el sangramiento, la infección de la herida operatoria, el tiempo de ventilación mecánica, uso de fluidos, vasodilatadores, morfina y antibióticos, la presión arterial y las alteraciones cardiacas, reduce el tiempo de hospitalización y sus costos. El calentamiento durante el intraoperatorio es suficiente para prevenir la hipotermia de forma efectiva, tanto en esta etapa como en el postoperatorio. Específicamente la aplicación del sistema de calentamiento con aire forzado es el método más efectivo en la mantención de la normotermia perioperatoria. 


\section{SUGERENCIAS}

Esta revisión deja en claro que los efectos del calentamiento son beneficiosos, por lo tanto se sugiere aplicar en toda persona adulta sometida a cirugía de más de una hora de duración, durante la etapa intraoperatoria (desde el ingreso de la persona al pabellón hasta el final de la cirugía), utilizando el sistema de aire caliente forzado, ya que se ha demostrado que es el más costo-efectivo. Además se recomienda la creación de una guía clínica en Chile respecto a la prevención y el manejo de la hipotermia perioperatoria, que considere los siguientes aspectos: 1) Identificación de antecedentes mórbidos y factores de riesgo de hipotermia. 2) Identificación del tipo de cirugía, ya que de ella depende el momento de aplicación del calentamiento y la ubicación del dispositivo. 3) Valoración de la temperatura corporal en las tres etapas del periodo perioperatorio. 4) Valoración de signos y síntomas de hipotermia como escalofríos, temperatura menor a $36^{\circ} \mathrm{C}$, piloerección y extremidades frías. 5) Aplicación de los sistemas de calentamiento disponibles, de preferencia aire forzado. 6) Información a la persona respecto al dispositivo de calentamiento, antes de su instalación. 7) Valoración del uso correcto del dispositivo de calentamiento (ubicación de la frazada y funcionamiento del dispositivo). 8) Capacitación al personal respecto a las características de los dispositivos de calentamiento disponibles y su uso correcto. Finalmente se sugiere investigar respecto a la magnitud de la hipotermia perioperatoria en Chile, ya que no se encontraron estudios en nuestra población.

\section{REFERENCIAS}

1. Rincón D, Sessler DI, Valero F. Complicaciones de la hipotermia transoperatoria. Rev Col Anest. 2004; 32(3):185-193.
2. Sessler DI. Complications and treatment of mild hypothermia. Anesthesiology. 2001; 95 (2):531-43.

3. Cooper S. The effect of preoperative warming on patients' postoperative temperatures. AORN J. 2006; 83(5):10731084.

4. Good K, Verble J, Secrest J, Norwood B. Postoperative hypothermia- the chilling consequences. AORN J. 2006; 83(5):1055-1066.

5. Leslie K, Sessler DI. Perioperative hypothermia in the high risk surgical patient. Best practice \& research clinical anaesthesiology. 2003; 17(4): 485-498.

6. Scott E, Buckland R. A Systematic Review of Intraoperative Warming to Prevent Postoperative Complications. AORN J. 2006; 83(5):1090-1113.

7. Testart R. Hipotermia perioperatoria en el paciente pediátrico. Rev anestesia [revista on-line] 2002. Hallado en: http://www.socanestesia.cl/rev_anestesia/0202/hipotermia.asp. Acceso el 14 de septiembre de 2002.

8. Quintana F, López E. Termorregulación y anestesia. En Compendio de anestesiología para enfermería. $3^{a}$ ed. Madrid: Hardcourt; 2001.

9. Kurz A. Prevention and treatment of perioperative hypothermia. Curr Anaesth Crit Care. 2001; 12(2): 96-102.

10. American Society of Perianesthesia Nurses. Clinical Guideline for the Prevention of unplanned perioperative hypother-

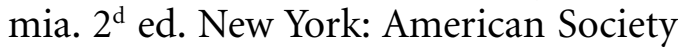
of perianesthesia nurses; 2001.

11. D'Angelo S, Cerqueira J, Pinheiro N, Amorim R, Rodrigues G. Preoperative Combined with Intraoperative SkinSurface Warming Avoids Hypothermia Caused by General Anesthesia and Surgery. J Clin Anesth. 2003; 15(2): 119125.

12. Fossum S, Hays J, Henson M. A comparison study on the effects of prewarming patients in the outpatient surgery setting. 
J Perianesth Nurs. 2001; 16(3):187-94.

13. Leaper D, Melling A. Antibiotic Prophylaxis in Clean Surgery: Clean Non -Implants Wounds. J Chemother. 2001; 1(13): 96-101.

14. Lenhardt R, Seybold T, Kimberger O, Stoiser B, Sessler D. Local warming insertion of peripheral venous cannulas: single blinded prospective randomized controlled trial and single blinded randomized crossover trial. BMJ. 2002; 325: 409-412.

15. Melling A, Ali B, Scott E \& Leaper D. Effects of preoperative warming on the incidence of wound infection after clean surgery: a randomized controlled trial. Lancet. 2001; 358: 876-880.

16. Young J, Shinn $H$, Jun $Y$, Woo Y, Jeong H, Lan Y. The effect of skin surface warming during anesthesia preparation on preventing redistribution hypothermia in the early operative period of off-pump coronary artery bypass surgery. Eur J Cardiothorac Surg. 2006; 29: 343-347.

17. Cavallini M, Baruffaldi F, Casati A. Effects of Mild Hypothermia on Blood Coagulation in Patients Undergoing Elective Plastic Surgery. Plast Reconstr Sur. 2005; 116:316-321.

18. Hasankhani H, Mohammadi E, Moazzami F, Mokhtari M, Naghizadeh M. The effects of warming intravenous fluids on perioperative haemodynamic situation postoperative shivering and recovery in orthopedic surgery. British Journal of Anaesthetic \& Recovery Nursing. 2005; 6(1): 7-11.

19. Lee L, Leslie K, Kayak E, Myles P. Intraoperative patient warming using radiant warming or forced-air warming during long operation. Anaesth Intensive Care. 2004; 32: 358-361.

20. Bock M, Muller J, Bach A, Bohrer H, Martin E, Motsch J. Effects of preinduction and intraoperative warming during major laparotomy. Br J Anaesth. 1998; 80 (2):159-63.
21. Mahoney C, Odom J. Maintaining intraoperative normothermia: a metanalysis of outcomes with costs. AANA J. 1999; 67:155-164.

22. Hohn L, Schweizer A, Kalangos A, Morel D, Bednarkiewicz M, Licker M. Benefits of intraoperative skin surface warming in cardiac surgical patient. Br J Anaesth. 1998; 80: 318-323.

23. Frank SM, Fleisher LA, Breslow MJ, Higgins MS, Olson KF, Kelly S, et al. Perioperative maintenance of normothermia reduces the incidence of morbid cardiac events: a randomized clinical trial. JAMA.1997; 227(14):1127-34.

24. Fleisher L, Metzger SE, Lam J, Harris A. Perioperative cost-finding analysis of the routine use of intraoperative forced air warming during general anesthesia. Anesthesiology. 1998; 88:1357-1364.

25. El-Rahmany H, Frank S, Schneider G, ElGamal N, Vannier C, Ammar R, Okasha $\mathrm{S}$. Forced air warming decreases vasodilator requirement after coronary artery bypass surgery. Anesth Analg. 2000; 90: 286-291.

26. Melling A, Leaper D. The impact of warming on pain and wound healing after hernia surgery: a preliminary study. J Wound Care. 2006;15: 104-108.

27. Pathi V, Berg G, Morrison J, Cramp G, McLaren D, Faichney A. The benefits of active rewarming after cardiac operations: a randomized prospective trial. J Thorac Cardiovasc Surg.1996; 111: 637641.

28. Kurz A, Sessler DI, Lenhardt R. Perioperative normothermia to reduce the incidence of surgical-wound infection and shorten hospitalization. N Engl J Med. 1996; 334: 1209-15.

29. Fong S, Cheng S, Khiam H, Poh Y, Yiong $\mathrm{H}$, Biauw C. A comparative study of three warming interventions to determine the most effective maintaining perioperative normothermia. Anesth Analg. 2003; 96: 171-176. 